to the naked eye. This property is of the greatest utility, not caly in mountaineering, but also in other pursuits which necessitate correct oricntation under foggy conditions. In order to obtain as large a visual field as possible, the glass, instead of being the usual round or oval shape, was triangular in form, thus giving an increased field of vision downwards and laterally.

The goggles described above have had a thorough and prolonged practical trial. They were used by the whole of the recent Everest expedition, which numbered, with porters, about ninety persons. Conditions were particularly scvere, and it was difficult, if not impossible, to remove the goggles for the purpose of wiping them when the men were fully dressed for climbing. Whereas on previous expeditions cases of snow-blindness arose, in this attempt there was not a single case. Since, in climbing, snow-blindness is more likely to occur when continual efforts have to be made in glancing downwards at the feet, a goggle which extends the field of vision in a- downward direction is of particular advantage. The extra field given by the "full-field" shape of the eyecups in the Everest goggle fulfilled this purpose, and rendered it unnecessary for the climber to remove the goggle in order to search for a good foothold. In all, the expedition was supplied with 200 pairs of goggles by Messrs. Hamblin Ltd. The same goggle has been extensively tried by motorists, who report better visibility in fog and great reduction in glare from the lights of oncoming traffic. This, added to the fact that the visibility is not sensibly affected, gives them a great advantage over other forms of " anti-glare" goggles.

\section{Protection in Industry}

The ill-effects of infra-red rays are well exemplified by " glass-blower's cataract." Glass-blowers, tinplate roller men, welders, and others working under similar conditions are all subject to this disease. In certain trades the process involves the production of ultra-violet light in addition to infra-red rays. In industry the type of glass must be varied to meet the needs of each particular kind of work. Thus the welder will require a different type of glass from the grindstone worker, who does not need the elimination of any part of the spectrum. While it is the simplest matter to devise protective goggles, as is evidenced by the vast selection already on the market, it is the most difficult thing in the world to induce workmen to wear them. This unwillingness can be attributed mainly to the serious defects in present-day glasses. These defects, which render the most scientifically efficient goggle useless from a practical point of view, are: (1) curtailment of the field of vision; and (2) poor ventilation, a truly definite handicap to a worker in a warm temperature. Poor ventilation not only reduces the comfort with which the eyes are used, but also reduces vision and the possibility of doing fine and accurate work through steaming of the glass.

For protection the goggles must be light, must be adapted in their transmission of visible light to the particular process in hand, and must have maximum ventilation and a maximum field of vision. These criteria can be obtained in the goggles under consideration above, fitted with a glass different from that supplied to the mountaineering expedition. Furthermore, they can be manufactured at a price which is very moderate. A heavy goggle cannot be worn with comfort for long. If too much of the visible spectrum be excluded, workers on piece rates lose in wage-earning capacity, and those engaged in accurate work in efficiency. The cooler the goggle the better it is in the estimation of the worker, and the more likely to appear to him to give adequate protection, since he judges the bad effects of heat rays mainly by cutaneous sensation, even although this be mainly produced by bad ventilation. The usual form of roundglassed goggle restricts the field, and consequently mobility; those providing a fuller field will be of greater usefulness, and more readily worn by workers. Perhaps not all the damage is done by the infra-red rays; some blame may attach to the ultra-violet radiations. Full protection, therefore, is only obtained from a glass which prevents rays from both the ultra-violet and the infra-red ends of the spectrum from reaching the eye.

Experiments were performed with laminated protective glass containing a congenial tint and a deposit of silver forming a mirror. It was found that the combination of the tint and the silver eliminates the defects present in each singly. The final glass, of which the analysis in the infra-red region is shown in Fig. 2, permits better visual acuity than the welder's glass in common use and that used by operators in ultra-violet light clinics, since it only reduces $6 / 4$ vision to $6 / 9$ (monocular). The depth of silvering can easily be varied to suit the requirements of different occupations, certain trades requiring no silvering or tinting at all, but only the protective lamination. With regard to ventilation, rather more can be obtained by a modification of the lower part of the goggle, a feature now being perfected. The maximum coolness will be given without seriously reducing the amount of protection afforded, by removing the sides of the eye-cup above, laterally, and below. The glass is eminently suited to the prevention of flying particles from entering the eyes. The silver mirror, protecting from infra-red by reflection rather than by absorption of the glass, should ensure the maximum coolness of these goggles. Finally, the spring fitting and the enlarged field should render the goggles as comfortable and as useful as is possible.

BibliogRAPHY

Lawson, Sir Arnold: "Tints and their Value," Iroc. Rov. Soc. Med November, 1924

Duke-Elder, W. S.: hecent Advances in Ophthalmology, second edition, 1929

Russell and Russell: Vltra-ivolet Radiation and Actinotherapy, 1927

Ormond, A. W.: " Notes Regarding the Pathological Effects of the Visible and Invisible Spectrum on the Eye," Guy's Hospital Reports, 1921, 1xxi, 193

\section{THE PROGNOSIS OF SYMPTOMLESS GLYCOSURIA}

\section{P. J. CAMMIDGE, M.D.}

In many cases of glycosuria the presence of the classical symptoms of diabetes mellitus, thirst, polyuria, and wasting, or the onset of one or other of the complications to which diabetics are liable, is an unmistakable indication of the nature of the disease, but in others the existence of sugar in the urine is discovered accidentally, and clinical symptoms attributable to defects of carbohydrate metabolism are lacking.

The prognosis in cases of the latter type is always difficult to decide, yet it is often a matter of serious importance, since a considerable proportion are detected in the course of a routine examination for life insurance, upon which much may depend. Biochemical tests may be helpful in some instances, but for the majority experience is the only available guide, and as statistical evidence is very scanty, differences of opinion are liable to arise, maybe to the detriment of the patient. Seeing that I have now records extending over more than thirty years, the following data, originally prepared for a discussion on glycosuria by the Assurance Medical Society, may help to fill the gap. 


\section{Review of Material}

Taking a series of 1,200 cases of which I have complete clinical and chemical records, I find that 780 were of the classical diabetic type and that in 420 the glycosuria was discovered accidentally, either in an examination for life insurance or in the course of a routine test of the urine for other purposes. Dividing the symptomless cases into groups according to the frequency and severity of the glycosuria, we have, first, a group of 69 cases in which sugar was found on only one occasion (uni-occasional); secondly, a group of 248 in which sugar was passed intermittently, and a third group of 103 with persistent glycosuria. All the patients in the uni-occasional group, some of whom have been under observation for fifteen or sixteen years, are still alive. The amount of sugar passed was always small, and, in some instances, was apparently due to a nervous cause. In the intermittent group the glycosuria was slight or moderate-that is to say, under 20 grams in the twenty-four hours-in 105 , and severe-more than 20 grams in the twenty-four hours-in 21. Of those with moderate glycosuria 9 have died -2 of malignant disease, 1 from cardiovascular disease, 1 from pneumonia, 1 from tuberculosis, 1 from acute pancreatitis, 1 from disease of the liver, 1 from an accident, and another, with a history of glycosuria for eighteen years, from old age at 81 . None of these cases have developed to such an extent as to call for the use of insulin, and all have been kept under control by dieting. Of the 21 cases of severe intermittent glycosuria, 1 has required insulin, and 3 have died-1 case from heart disease, 1 from pucumonia, and 1 following an operation. Moderate persistent glycosuria was found in 43 cases, and 3 of these have died-1 from malignant disease and 2 from cardicvascular disease; of this group 1 patient is now on insulin. Severe persistent glycosuria was met with in 36 cases, and 13 of these are now taking insulin regularly, while 11 have cied-4 from cardiovascular disease, 3 from septicaemia, 1 from malignant disease, 1 from diabetic coma, 1 from an accident, and 1 committed suicide. It will thus be seen that out of 420 cases of symptomless glycosuria 26, or 6 per cent., have died, although death was due to diabetic coma in only one instance.

A more satisfactory way to study the influence of symptomless glycosuria on longevity is to divide the cases in decades, according to the age when sugar was discovered, and consider them from the point of view of the sugar content of the blood. Of the 420 cases under review 172 gave a normal, or subnormal, blood sugar curve after atest meal of glucose. Twenty-three were under 10 years

age ; in 5 the glycosuria was uni-occasional, in 16 intermittent, and in 2 persistent. Twenty were aged from 11 to 20 years, and in 5 sugar was found only once, in 11 it was intermittent, and in 4 persistent. Twenty-one fell in the next decade ; 5 showed uni-occasional, 12 inter. mittent, and 4 persistent, glycosuria. Forty-one patients were between 31 and 40 years of age ; of these 5 passed sugar once; in 33 there was intermittent, and in 3 persistent, glycosuria. Thirty-three were batween 41 and 50 years of age ; 4 were uni-occasional, 23 intermittent, and 6 persistent glycosurias. Twenty-one were between the ages of 51 and 60 , with 1 uni-occasional, 15 intermittent, and 5 persistent glycosurias. Over the age of 60 there were 13 cases, 1 being uni-occasional and 12 intermittent. A moderate amount of sugar-under 20 grams a day - was passed by all the patients in this group except 2-1 in the third and 1 in the fifth decade; but all are alive and well, and most are taking a normal diet, some with added glucose to prevent the hypoglycaemic symptoms from which they suffer. The other 248 cases showed a more or less pronounced hyperglycaemic blood sugar curve after a test meal of glucose when they were first seen.

\section{Cases by Age Groups}

Considered in decades, the noteworthy points which emerge are as follows :

First Decade.-There were $\mathbf{8}$ cases in this group, all of moderate severity. One was discovered accidentally in a routine examination, 7 were the children of glycosuric patients, and their urine and blood were investigated as a consequence. Six are now on a normal diet; 2 where sugar was present practically from birth are on restricted diets, but are alive at the ages of 13 and 15 years respectively.

Second Decade.-In this decade there were 16 cases14 moderate and 2 severe. Two have died, one of the mild intermittent type, from heart disease, after thirtyfive years, the other, a severe persistent case, after twenty-four years from septicaemia.

The second patient was accidentally found to be passing sugar when she was 14 years of age, but apparently suffered no ill effect until she became pregnant at the age of 21 ; she was then gravely ill, and the pregnancy was terminated. Two subsequent pregnancies were attended by less grave metabolic disturbance, and went to term. In the intervals, and after the last pregnancy, the patient refused to diet, and lived a normal life; eventually a carbuncle developed and gave rise to general septicaemia, from which she died, in spite of the free use of insulin.

Two other cases, both females, are of interest, for they were hyperglycaemic when first seen, but after a period of dieting were gradually able to resume a normal diet; now they are hypoglycaemic and are taking daily doses of glucose, one ten years and the other thirteen years after the onset of the glycosuria. Other cases in this group have passed sugar for sixteen, nineteen, and twenty-cne years, and are apparently in good health, without the use of insulin.

Third Decade.-Of the 34 cases in this decade, 29 had moderate and 5 severe glycosuria. One has died from cardiovascular disease, at the age of 70 , after being under treatment for mild glycosuria for forty years. All the others are still alive, one thirty-six years and another thirty-two years after the discovery of the glycosuria. One patient, after sixteen years' treatment by diet alone, is now taking insulin.

Fourth Decade.-In this decade there are 45 cases, 32 moderate and 13 severe, with two deaths, both from accident; one, a mild case, was killed in an aeroplane crash, and the other, a severe case, in a motor accident. Five are now on insulin, one of these after being treated by diet for twenty-three years, and others for shorter periods. Dietetic treatment has kept the disease under control for twenty-eight and thirty years in other cases in this decade.

Fifth Decade.-The number of patients falling into this group is 56 , and of these 41 were moderate and 15 were severe cases. Five of the former and 3 of the latter have died. Three of the moderate cases died of malignant disease, one three months, another twelve months, and the third eighteen months after the glycosuria was discovered ; a fourth patient died at the end of a year from disease of the liver, and a fifth from tuberculosis after fifteen years. The three severe cases succumbed, to cardiovascular disease after seventeen years, septicaemia after eighteen years, and pneumonia after twenty-four years, respectively. None of the patients in this decade were treated with insulin.

Sixth Decade.-In this decade there were 62 cases. Out of 44 of the moderate type 3 have died-1 from acute pancreatitis, after two years, 1 from pneumonia, after six years, and 1 from heart disease, after eleven years. Of the 18 severe cases 5 have died--1 following an operation, after eleven years, 1 from septicaemia following carbuncle after twenty years, 2 from carciovascular disease after 
twenty and twenty-three years respectively, and 1 from malignant disease after twenty-two years. The lastmentioned, and one of the heart cases, was treated with insulin for five years. Four others in this decade who are still alive are taking insulin.

Sixty Years of Age and Over.-Of the 29 cases 24 were moderate and 5 severe. One of the moderate cases died after eighteen years' treatment at the age of $\mathbf{s} 1$. Four of the five severe cases are also dead ; one committed suicide within a week of the discovery of his glycosuria, one died of heart disease after three years' dietetic treatment and three on insulin, another died of diabetic coma after two years' diet and five years on insulin ; the fourth, after eleven years' diet, died from heart disease. The fifth, a severe case, is now on insulin.

\section{Pseudo-glycosuric Cases}

In addition to the cases of symptomless glycosuria already considered I have records of a number of patients who were referred to me because their urine gave a positive reaction for sugar, but further investigation showed that this was due to some other reducing substance than dextrose. Taking 200 of these, I find that in 63 the sugar was true laevulose, in 67 it proved to be pseudo-laevulose (iso-glycuronic acid), 30 were cases of pentosuria, 11 being of the true or essential type and 19 of the alimentary variety ; 16 passed lactose, and were all either pregnant or lactating; in 16 the reducing action of the urine was due to an excess of glycuronic acid; cne patient, a middle-aged man who was consuming very large quantities of milk, passed galactose ; and another, who was a heavy beer-drinker, excreted maltose. Although these cases are not, strictly speaking, examples of "symptomless glycosuria," they have some bearirg on the question under discussion, since the response given $b_{y}^{\prime}$ the urine to the ordinary reduction tests for sugar resulted in an incorrect diagnosis of glycosuria being made, in the first instance, and in all the classical symptoms of diabetes were lacking. The abnormal condition cf the urine was discovered accidentally in every case, some during examination for life insurance. Several of the cases of pentosuria and laevulosuria had been on antidiabetic diets for considerable periods before they came under my care.

The more thoroughly the family history of cases of glycosuria is investigated the more evident does it become that heredity plays an important part in the aetiology of the condition, and it would seem probable that the way in which the abnormality is inherited, as a familial or direct character, has some bearing on its severity, and therefore on the prognosis. In a series of 800 cases of diabetes mellitus I published in 1928 (Journal, 1928, ii, 738), 28 per cent. were found to give an ancestral or family history of the disease, but in the present series the proportion is 38 per cent., taking the classical diabetics alone, or, including the symptomiess glycosurias, 36.5 per cent. Considering the 420 cases of symptomless glycosuria separately, a history of glycosuria in a blood relation was obtained in 142 (33 per cent.). Of the 248 who showed a hyperglycaemic curve after a test meal of glucose, 88 (35 per cent.) gave a family history of glycosuria, while a positive history was obtained in 54 out of 172 (31 per cent.) of those who gave a normal or subnormal blood sugar curve. A dominant, or direct, history was found in 126 (16 per cent.), and a recessive, or familial, history in 171 (22 per cent.) of the classical diabetic cases; but in the symptomless glycosurias there were 124 (29 per cent.) dominant and only 18 (4 per cent.) recessive. Of the eighteen symptomless cases with a recessive family history 15 were of the severe persistent type, and gave a hyperglycaemic curve after a test meal of glucose. All required treatment with insulin-although not until after the lapse of some years in most instancesand two have died, one from malignant disease of the colon twenty-two years later, and the other from heart disease after six years' treatment. The other three gave hypoglycaemic blood sugar curves with a moderate amount of urinary sugar, and are now living normal lives.

\section{SUMMARY}

These results seem to suggest that:

1. The prognosis in symptomless glycosuria is usually good.

2. Most patients live for many years after the onset of the glycosuria, although limitation of the diet, and eventually insulin, may be necessary in some.

3. Death is generally due to some intercurrent condition, but complications such as septic infection, pregnancy, and surgical operations are liable to increase the metabolic disturbance, and may bring about a fatal termination.

4. Patients with a recessive (familial) history are more likely to develop a severe glycosuria, and require the use of insulin, than those with a dominant (direct) family history, which is the most common type.

\section{Memoranda MEDICAL, SURGICAL, OBSTETRICAL}

\section{NEMBUTAL IN ETHER CONVULSIONS}

I am tempted to put the following case of ether convulsions on record because, in an exhaustive search of the literature, I have been unable to find any case in which either spinal anaesthetic or nembutal has been used as a form of treatment. In the literature as a whole there is an absence of any definite recommendation to treatment, but it reveals a very high mortality rate. There is no doubt that the case here recorded was a typical example of the condition, and one likely to have proved fatal.

The patient, a well-developed boy aged 12 , was admitted to the Prince of Wales's Hospital on June 19th, 1933, with a history of four days of abdominal pain, localizing finally in the right iliac fossa, together with vomiting and constipation. There had been a marked intermittency in the symptoms during the four days. His previous history contained nothing of note, except "sunstroke" at the age of 2 for one week, "nightmares" attributed to threadworms from the age of 5 to 8 , and a fall on the head producing a wound requiring suture at the age of 10 . There was no history of fits, petit mal, chorea, enuresis, or asthma. The boy was one of a healthy family of three; no blood relatives had suffered from epilepsy, and there was nothing significant in the family history.

Examination of the patient showed that he was very toxic the tongue being dirty and breath offensive. Respiration was mainly thoracic, the temperature $101^{\circ} \mathrm{F}$., and the pulse 120. The abdomen was gencrally rigid, and there was marked tenderness over the right lumbar region and iliac fossa. Rectal examination revealed peritoneal tenderness Lungs, heart, and urine appeared normal. A diagnosis was made of acute obstructive appendicitis with peritonitis, with the appendix probably in the retrocaecal position.

\section{OpERATION}

After a hypodermic injection of 1/100 gram of atropine the patient was taken to the theatre. A heat-wave was in progress, and the temperature of the theatre was $85^{\circ} \mathrm{F}$. (the combination of a hot day and a toxic patient seems to be favourable for ether convulsions). The patient was anaesthetized first with ethyl chloride and then by open ether The anaesthetic was not satisfactory; the patient did not settle down, and was breathing in a shallow and jerky manner, with the result that he was cyanosed most of the time. General peritonitis was present, and a gangrenous 